\title{
¿Cómo se define un problema público? La redefinición del problema en las políticas de bienestar locales. El caso del Plan de Acción de Servicios Sociales de la Diputación de A Coruña ${ }^{1}$
}

\author{
How to define a public problem? The problem redefinition in local \\ welfare public policies. The Social Services Action Plan of the \\ provincial Government of A Corunna
}

\author{
Manuel Joaquín Pérez Castreje \\ Nova Works Consulting S. Coop. Galega (España) \\ ORCID: https://orcid.org/0000-0003-2531-0016 \\ manueljoaquin.perez@rai.usc.es
}

\section{NOTA BIOGRÁFICA}

Doctor en Ciencias Políticas (USC) y Máster en Gestión Pública (ESADE-UAB-UPF), desarrolla su carrera en el campo del análisis de políticas públicas, especializándose en las políticas de bienestar a nivel local y autonómico, y colaborando en auditorías y evaluaciones de programas internacionales. En los últimos años ha coordinado la elaboración del Plan de Acción de Servicios Sociales de la Diputación de A Coruña (20172020), así como trabajos de desarrollo de la economía social y de mejora organizativa en entidades locales.

\section{RESUMEN}

El artículo analiza el proceso de redefinición del problema público relacionado con las políticas de bienestar locales llevado a cabo a través del Plan de Acción de Servicios Sociales de la Diputación de A Coruña, así como los elementos básicos que determinaron la mencionada redefinición y el abanico de medidas que integran el Plan. La complementariedad de la aplicación del marco analítico que ofrecen el análisis de políticas públicas y el neo-institucionalismo permite observar la importancia de los valores del grupo promotor como actor central en el diseño del Plan a la hora de incorporar una visión multinivel, lo cual supone una evolución no incremental en el modelo de política pública de bienestar liderado por la institución provincial en su colaboración con los Ayuntamientos menores de 20.000 habitantes. Además, el artículo incorpora el debate sobre las posibles dificultades y/o desvíos que se pueden presentar debido a la necesaria incorporación de nuevos actores en el proceso de implementación del Plan.

\section{PALABRAS CLAVE}

Bienestar; multinivel; política pública; definición; problema público.

\section{ABSTRACT}

The article deals with the public problem redefinition process related to local welfare public policies carried out through the Social Services Action Plan by the provincial Government of A Corunna, as well as pointing

1 El Plan de Acción de Servicios Sociales no se habría realizado sin el apoyo y colaboración del personal del Servicio de Acción Social de la Diputación de A Coruña, liderados por Ánxela Franco, Diputada Delegada de Bienestar (2015-2019). Sirvan estas líneas para reconocer y agradecer su colaboración e impulso. El agradecimiento se extiende a los/las evaluadores anónimos y a la editora de la Revista, cuyas sugerencias han mejorado sustancialmente la primera versión del artículo. 
out the crucial elements that determinate its redefinition and the wide range of measures included in the Plan. Complementarity between policy analysis and neo-institutionalism analytical frames allow us to observe the values of the advocacy group as the central actor in the Plan's design and its effect when incorporating a multilevel view, which implies a non incremental evolution in the local welfare public policy lead by the provincial Government and implemented in municipalities under 20.000 inhabitants. Moreover, the article embraces the discussion about the difficulties and/or deviations that may arise, due to the inevitable integration of new actors along the implementation process of the Plan.

\title{
KEYWORDS
}

Welfare; multilevel; public policy; definition; public problem.

\begin{abstract}
SUMARIO
1. INTRODUCCIÓN. 2. EL MARCO ANALÍTICO. 3. EL ESTUDIO DE CASO. EL PLAN DE ACCIÓN DE SERVICIOS SOCIALES. 3.1. LA NECESIDAD DEL PLAN DE ACCIÓN Y SUS FUNDAMENTOS. EL NUEVO ROL DE LA DIPUTACIÓN. 3.2. LA METODOLOGÍA DE LA ELABORACIÓN DEL PLAN. LOS VALORES Y LAS CREENCIAS. 3.3. LOS DOCUMENTOS INTEGRANTES DEL PLAN. 3.3.1. EI Marco Estratégico. 3.3.2. Los Diagnósticos. Las agendas locales del bienestar. 3.3.3. El Plan de Acción. 3.3.4. Las limitaciones del Plan de Acción. 4. LA EVOLUCIÓN DE LA DEFINICIÓN DEL PROBLEMA PÚBICO Y SUS EFECTOS EN LA POLÍTICA. 5. CONCLUSIONES Y APORTACIONES DE LA INVESTIGACIÓN. REFERENCIAS BIBLIOGRÁFICAS.
\end{abstract}

\section{INTRODUCCIÓN}

Entre los años 2017 y 2020, el Servicio de Acción Social de la Diputación de A Coruña desarrolla un proceso de planificación estratégica en el que se analiza la situación de los departamentos municipales de bienestar de los Ayuntamientos de la provincia y del propio ente provincial, para la definición de las principales líneas de futuro a acometer desde la Diputación.

Los trabajos se llevan a cabo a través de un proceso participativo presencial en el que se integran la práctica totalidad de los equipos técnicos de bienestar de los Ayuntamientos menores de 20.000 habitantes y numerosas entidades sociales. Como consecuencia, la Diputación cuenta con un conjunto de informes en los que se estudia a nivel provincial, comarcal y local la situación de las políticas de bienestar; y en los que se diseña un nuevo marco de actuación. El Plan supone una importante evolución de la política pública desarrollada hasta el momento.

El Plan de Acción no sólo se centra en el trabajo llevado a cabo desde la Diputación en su rol de apoyo a los entes locales, sino que estudia los factores determinantes de las políticas locales de bienestar. El Plan entiende que unas reglas institucionales que mejoren la relación entre las administraciones incidirán positivamente en el rendimiento de la política y, por lo tanto, en la prestación de servicios a la ciudadanía.

El artículo tiene como objetivo recoger, desde la perspectiva del análisis de políticas públicas, la redefinición del problema público presente en el Plan de Acción con respecto al modelo previo de política, y cómo esta variación determina las nuevas medidas de la política pública. En este sentido, a lo largo del texto nos preguntaremos ¿en qué se centraba el anterior modelo de política y cuál era el problema que pretendía resolver? ¿Cómo se gesta el Plan de Acción? ¿Qué actores participan en su elaboración? ¿Cuál es la nueva definición del problema? ¿Cuáles son las principales características de la nueva política? La aplicación del modelo de análisis de políticas públicas de Subirats et al. (2008) permite responder a las preguntas planteadas y al objetivo del artículo. En el modelo, la definición del problema se considera el elemento inicial a partir del cual se construyen los instrumentos de intervención diseñados para superar la distancia entre la situación observada y la situación deseada.

El artículo se inicia aportando una perspectiva sobre el modelo de análisis de políticas públicas y sobre los conceptos a utilizar. En el de estudio caso se analiza la necesidad y oportunidad del Plan de Acción, la metodología utilizada para su elaboración y sus principales hitos y limitaciones para, a continuación, analizar la evolución en la definición del problema público acometida. Finalmente, a modo de conclusiones, se responde a las preguntas realizadas, y se exponen los principales resultados extraídos de la elaboración del Plan para su posible influencia en otras políticas multinivel. 
GAPP. Nueva Época - N. ${ }^{\circ}$ 27, noviembre 2021 - ISSN: 1989-8991 - DOI: https://doi.org/10.24965/gapp.i27.10940 - [Págs. 85-100] ¿Cómo se define un problema público? La redefinición del problema en las políticas de bienestar locales. El caso del Plan de Acción..

Manuel Joaquín Pérez Castreje

\section{EL MARCO ANALÍTICO}

En el artículo se utiliza el modelo de análisis de políticas públicas de Subirats et al. (2008), complementándolo con el neo-institucionalismo de March y Olsen (1997), que aporta amplitud y profundidad explicativa al análisis y a las relaciones de causalidad propias de la política.

El modelo diseñado por Subirats et al. (2008) es un marco analítico en fases que representa el ciclo de una política, siguiendo la tradición de numerosos autores (Bardach, 1998; Dye, 1992; Jones, 1970; Laswell, 1951, Laswell, 1971; Meny y Thoenig, 1992; Parsons, 1995). De todos modos, las fases y el ciclo de una política son una elaboración conceptual y una representación simplificada que no siempre se ve representada de modo secuencial en la realidad (Lindblom, 1991; Dye, 1992), por lo que se asumen sus limitantes, ya que la política empírica se considera un flujo de decisiones y procedimientos a los que se intenta dar sentido (Muller, 2002).

FiguRA 1. EL MOdELO dE ANÁLISIS DE POLÍTICAS PÚBLICAS

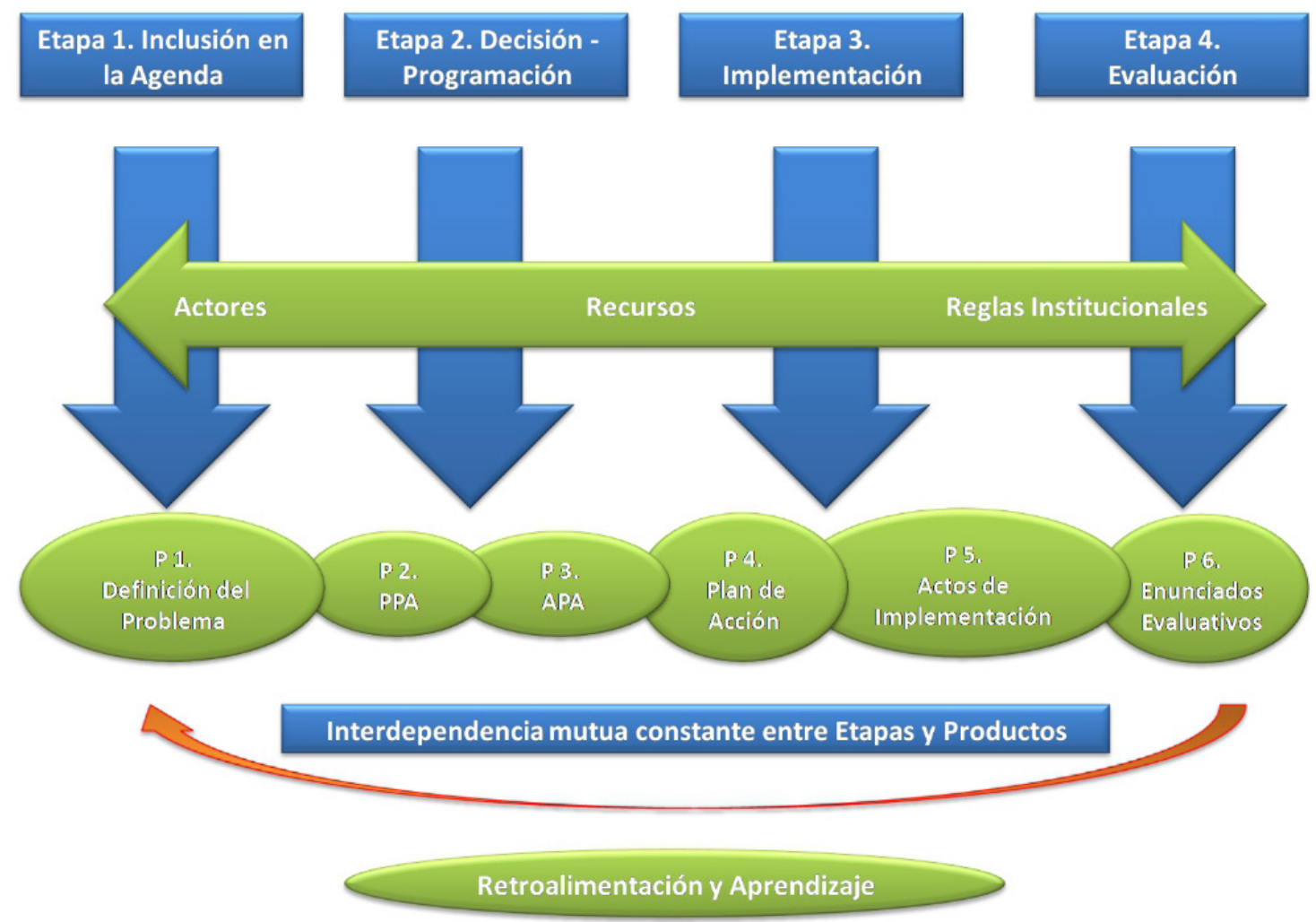

Fuente: Pérez (2020) a partir de Subirats et al. (2008).

El modelo se compone de etapas que, en su interior, contienen productos intermedios entendidos como el resultado de las propias etapas. Así, la etapa 1: inclusión en la agenda contiene el producto intermedio 1: definición del problema público; la etapa 2: decisión y programación tiene como resultados el producto intermedio 2: programa de actuación político-administrativo, y el producto intermedio 3: acuerdo políticoadministrativo; la etapa 3: implementación se integra por el producto intermedio 4: plan de acción y por el producto intermedio 5: actos de implementación; y finalmente la etapa 4: evaluación tiene como resultado el producto intermedio 6: enunciados evaluativos.

Las etapas y productos intermedios son el resultado de la interacción y el juego de (i) los actores; (ii) las reglas institucionales; y (iii) los recursos que los actores movilizan. La política es el resultado construido de la interacción entre estos elementos, por lo que representa la distribución del poder en la sociedad. Así, el objeto del análisis de las políticas públicas «no es el poder político como tal, sino su utilización para resolver los problemas colectivos» (Subirats et al., 2008, p. 37). 
GAPP. Nueva Época - N. ${ }^{\circ}$ 27, noviembre 2021 - ISSN: 1989-8991 - DOI: https://doi.org/10.24965/gapp.i27.10940 - [Págs. 85-100] ¿Cómo se define un problema público? La redefinición del problema en las políticas de bienestar locales. El caso del Plan de Acción..

Manuel Joaquín Pérez Castreje

El artículo se centra en la redefinición del problema público realizada en el Plan de Acción de Servicios Sociales de la Diputación de A Coruña con respecto a la definición previa sobre la que se sustentaba el modelo anterior de la política. Como se puede observar en la Figura 1, la definición del problema es el elemento a partir del cual se construye la política, siempre teniendo en cuenta el dinamismo y la vinculación entre Etapas y productos intermedios.

En relación al problema público, Cobb y Elder (1984, p. 80), siguiendo a Wildawsky (1979) afirman que «los problemas son elaboración de los hombres. Siempre existen concepciones múltiples. No hay problemas definidos de modo único». En este sentido, la definición del problema público, para Stone (1989, p. 299), es la manipulación de las imágenes de las condiciones de la realidad por los actores que interactúan y compiten, de modo que estas condiciones reales se definen como problemas a través del retrato estratégico de las historias causales.

La definición del problema es parte de la política y su elaboración (o reelaboración) determina desde la presencia de actores involucrados en ella hasta las intervenciones posteriores. Esta construcción «... se vincula con las percepciones, representaciones, intereses y valores de los actores involucrados...» (Subirats et al., 2008, p. 128), de modo que son estos elementos internos los que influyen en el posicionamiento de los actores en relación a unos hechos y, por lo tanto, en la interpretación de los hechos como una situación a resolver.

En este sentido, los sistemas de creencias de los actores cuentan con un rol central en la interpretación de la situación como un problema y, posteriormente, en el diseño e implementación de programas. Así, estas creencias, de especial relevancia en las coaliciones promotoras o de causa (Sabatier, 1988; Sabatier, 1998; Sabatier y Jenkins-Smith, 1993; Sabatier y Weible, 2007), representan valores, relaciones causales, teorías implícitas relacionadas con las maneras de alcanzar los objetivos, o percepciones sobre los instrumentos a utilizar para alcanzar los mencionados objetivos.

Como se puede comprobar, las ideas (Lakoff, 2007) y los valores de los actores tienen un rol fundamental en la definición de un problema. Este enfoque enlaza con la potencialidad del neoinstitucionalismo que afirma que los valores, creencias y preferencias de los actores son aspectos endógenos a las instituciones, entendidas como «... un conjunto de reglas y rutinas interconectadas que definen las acciones correctas en términos de relaciones entre roles y situaciones» (Peters, 2003, p. 50). Así, «... las instituciones políticas definen el marco en el que tiene lugar la política» (March y Olsen, 1997, p. 65).

El neoinstitucionalismo permite analizar el comportamiento y la actividad de las instituciones desde la generación de rutinas que proveen filtros de interpretación $y$ que proporcionan estabilidad al restringir la incertidumbre (Hall y Taylor, 1996; March y Olsen, 1997). La estabilidad del entorno institucional refuerza (y es reforzada) a través de una cultura que proporciona visiones, identidad y mitos comunes (March y Olsen, 1997, p. 105) que, a su vez, determinan la percepción de los individuos y su modo de actuar.

El comportamiento institucional y de los actores e individuos que se integran en la institución se explican atendiendo a las reglas institucionales. Para Ostrom (2013, p. 54-55) las reglas institucionales son: «Un entendimiento, compartido por los participantes, acerca de las prescripciones obligatorias que indican qué acciones (o resultados) se imponen, se prohíben o se permiten». Estas son desarrolladas y aplicadas por un conjunto de actores para organizar actividades repetitivas que producen resultados que, en la medida de lo posible, son previsibles. Por su parte, Subirats et al. (2008, p. 98) definen las reglas institucionales como «estructuras y reglas formales explícitas y generalmente formalizadas jurídicamente, y también como normas informales implícitas, pero compartidas por los miembros de una organización o comunidad».

Las normas, valores y rutinas presentes en una institución generan el modelo de toma de decisiones conocido como lógica de lo apropiado (March y Olsen, 1997). En este modelo los actores e individuos que interactúan en la institución no deciden con el objetivo de maximizar preferencias, sino para ser congruentes con sus roles y responsabilidades que, a su vez, están definidos en las normas y tradiciones.

La estabilidad del comportamiento institucional genera un contexto histórico de elevada influencia en la definición de políticas públicas denominado path dependence o senda de dependencia (Gallego et al., 2003; North, 1990; Peters, 2003). La senda de dependencia crea inercia de actuación, limita alternativas y refuerza la estabilidad de la institución.

Frente a esta estabilidad, la evolución y el cambio institucional son complejos (Ayala, 1999; Hall y Taylor, 1996; Peters, 2003). De todos modos, frente a la tendencia conservadora de los procesos institucionales (March y Olsen, 1997, p. 120), aparecen posibilidades de cambio a través del path departure, entendido como la adaptación de las instituciones mediante la redirección de los principios a seguir (Adelantado, 2011; 
GAPP. Nueva Época - N. ${ }^{\circ}$ 27, noviembre 2021 - ISSN: 1989-8991 - DOI: https://doi.org/10.24965/gapp.i27.10940 - [Págs. 85-100] ¿Cómo se define un problema público? La redefinición del problema en las políticas de bienestar locales. El caso del Plan de Acción..

Manuel Joaquín Pérez Castreje

Ebbinghaus, 2005); o la sedimentación, en donde se generan capas sucesivas con nuevas reglas y normas que solventan disfunciones, lo que supone la evolución de la institución al tiempo que se asienta el modelo previo, pasando de la dependencia del pasado al concepto de rumbo (Peters, 2003, siguiendo a Tolbert y Zucker, 1996).

El marco teórico expuesto es utilizado para analizar la redefinición del problema público llevada a cabo en el Plan de Acción de Servicios Sociales, estudiando los factores que inciden en su redefinición y los efectos posteriores sobre la política.

\section{EL ESTUDIO DE CASO. EL PLAN DE ACCIÓN DE SERVICIOS SOCIALES}

\subsection{La necesidad del Plan y sus fundamentos. El nuevo rol de la Diputación}

El Plan de Acción se enmarca en el cambio del sistema de financiación que la Diputación de A Coruña pone a disposición de los Ayuntamientos. El nuevo sistema se implanta en el año 2017 y, en relación a las políticas de bienestar, supone la eliminación y modificación de diferentes convocatorias anuales de apoyo financiero.

A partir del año 2017 entra en vigor el Plan de Obras y Servicios (en adelante POS+) como principal sistema con el que la Diputación de A Coruña colabora en la financiación de los Ayuntamientos. El POS+ se basa en una distribución financiera establecida en relación a diferentes variables ${ }^{2}$, supone la desaparición de las convocatorias anuales de las líneas de apoyo financiero en varios ámbitos ${ }^{3}$, incluido en materia de bienestar ${ }^{4}$, y el fortalecimiento del FOPPSS (Programa de Financiación de los Servicios Sociales Comunitarios Municipales $)^{5}$ como principal línea de financiación de los departamentos municipales de bienestar. El FOPPSS aporta anualmente fondos para la cobertura del personal técnico de apoyo, personal administrativo y horas del Servicio de Ayuda en el Hogar (en adelante SAF).

En este contexto, el Servicio de Acción Social de la Diputación detecta la necesidad de contar con un instrumento de planificación que identifique los recursos, prioridades y futuras líneas de acción de las políticas de bienestar. Esta necesidad se cubre mediante el Plan de Acción, desarrollado entre los años 2018 y 2020.

Así, una coalición interna que actúa a modo de policy entrepreuneur o emprendedor de políticas (Kingdon, 1984) se erige como promotora (Sabatier, 1988; Sabatier, 1998; Sabatier y Jenkins-Smith, 1993; Sabatier y Weible, 2007) del Plan de Acción. Esta coalición se compone, desde la parte política, por la Diputada Delegada de Bienestar en la legislatura $2015-2019^{6}$ y, por la parte técnica, por el personal técnico de la sección. La coalición revisa la política de bienestar aprovechando la ventana de oportunidad (Aguilar, 2000; Kingdon, 1984) abierta por la conformación del gobierno por el Partido de los Socialistas de Galicia-PSOE y el Bloque Nacionalista Galego que implanta el POS+.

2 Véase Base 4. ${ }^{\text {a }}$ de las Bases reguladoras del Plan Provincial de Cooperación ás obras e servizos de competencia municipal «POS+ 2017». BOP núm. 226 de martes 29 de noviembre de 2016. Disponible en https://bop.dicoruna.es/bopportal/publicado/2016/11/29/2016_0000009792.pdf. Consultado en enero del 2021.

3 En el POS+ del año 2017 se integran 11 líneas de financiación (2 de cultura, 2 de deportes, 2 de promoción económica, 2 de

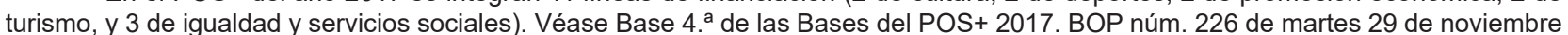
de 2016. Disponible en https://bop.dicoruna.es/bopportal/publicado/2016/11/29/2016_0000009792.pdf. Consultado en enero de 2021.

4 En 2016 las políticas de bienestar locales eran financiadas a través de 3 líneas: el FOAXCA (programa de mantenimiento de servicios sociales municipales), con 1.363.004,62€; (II) el FO063A (programa de envejecimiento activo), con 261.000€; y (III) el FOPPSS (programa de equipos y horas de SAF libre concurrencia en Ayuntamientos menores de 20.000 habitantes) con 4.020.149,78€.La financiación total asciende a 5.644.150,40€.

5 El presupuesto del FOPPSS pasa de 4.037.275,46€ en 2017 a 8 millones de euros en 2021. Véanse BOP núm. 39 de viernes 24 de febrero de 2017, en https://bop.dacoruna.gal/bopportal/publicado/2017/02/24/2017_0000001474.pdf y BOP núm. 215 de miércoles16 de diciembre de 2020, en https://bop.dacoruna.gal/bopportal/publicado/2020/12/16/2020_0000009507.pdf. Además, como consecuencia de la COVID-19, la Diputación asigna una partida específica en el POS+ para servicios sociales de 4 millones de euros en 2020 y 14 millones en 2021. Véanse para el 2020 la certificación de aprobación plenaria del 26 de junio del 2020 (https://www.dacoruna.gal/ files/5715/9679/0404/CERT_ASINADA_APROBACION_POSAD_I_2020.pdf) y para el 2021, BOP núm. 205 de martes 1 de diciembre de 2020 (https://bop.dicoruna.es/bopportal/publicado/2020/12/01/2020_0000008894.pdf) Consultados entre febrero y mayo del 2021.

6 En la legislatura 2015-2019 Ánxela Franco Pouso (BNG) es la Deputada Delegada de Igualdade e Benestar. Concelleira en Porto do Son (2003-2019) y Teniente Alcalde (2009-2011). Ver entrevista de Nós Diario titulada "O BNG veu á Deputación para cambiala", de 15 de febrero del 2018. Disponible en https://www.nosdiario.gal/articulo/politica/bng-veu-deputacion-cambiala/20180214164111066026. html. Consultada en febrero del 2021. 
GAPP. Nueva Época - N. ${ }^{\circ}$ 27, noviembre 2021 - ISSN: 1989-8991 - DOI: https://doi.org/10.24965/gapp.i27.10940 - [Págs. 85-100] ¿Cómo se define un problema público? La redefinición del problema en las políticas de bienestar locales. El caso del Plan de Acción..

El Plan de Acción, en su concepción y desarrollo por un equipo específico, supone un avance con respecto a la senda de dependencia y a la sedimentación (Peters, 2003 siguiendo a Tolbert y Zucker, 1996) de las reglas institucionales (Ostrom, 2013) de las políticas de bienestar locales lideradas desde la Diputación. Estas reglas tenían su herramienta más destacada en las convocatorias de líneas de apoyo financiero anuales, sin venir acompañadas de planificaciones o evaluaciones que superaran el seguimiento y control administrativo del ciclo de la subvención. Esta situación crea unas rutinas, unos valores y una lógica de lo apropiado (March y Olsen, 1997) en los que se enmarcan los actores de la política desde una perspectiva multinivel.

Además, la senda de dependencia provoca una limitación de los recursos humanos del departamento de bienestar de la Diputación y su focalización en el ciclo de la subvención que, a su vez, redunda en la escasez de los espacios de interacción con los equipos técnicos municipales. Este contexto dificulta la creación de un modelo sustantivo común de la política y limita la generación de confianza, aspecto clave para el trabajo en red (Metcalfe, 1993; Brugué et al., 2014).

El Plan de Acción se entiende como una oportunidad para revisar la política de bienestar, aplicando el concepto de path departure (Adelantado, 2011 siguiendo a Ebbinghaus, 2005) al modelo previo.

Dentro de esta revisión, el Plan de Acción asume una concepción de las políticas de bienestar basada en la interdependencia multinivel y la complejidad en un marco de gobernanza. El bienestar se enmarca dentro de las políticas de servicios a las personas considerándose como de «máxima confluencia multinivel» (Gomà y Subirats, 1998). En ellas, el rendimiento final para la ciudadanía depende del establecimiento de relaciones eficaces entre las administraciones competentes.

Este enfoque es diferente al modelo previo que, centrado en la utilización de las convocatorias de subvenciones como medio de relación entre las administraciones, genera una actuación basada en la distancia entre el ente financiador y el ente prestador de servicios.

El nuevo marco conceptual es coherente con la Ley 13/2008 de Servicios Sociales de Galicia, que en su Art. 6 establece las competencias de asistencia técnica, económica y jurídica a los Ayuntamientos como propias de las Diputaciones. Además, en el Art. 31 del Decreto 99/2012 se establece el apoyo prioritario de las Diputaciones en el financiamiento del SAF básico de los Ayuntamientos menores de 20.000 habitantes y en la contratación de personal técnico de los servicios sociales comunitarios.

La normativa recoge la colaboración entre las Diputaciones y los Ayuntamientos en relación a los servicios sociales comunitarios básicos y específicos, que son competencia municipal según el Título VIII de la Ley 13/2008 de Servicios Sociales de Galicia.

Los servicios sociales comunitarios básicos (Art. 9-11 de la Ley 13/2008) constituyen el acceso de la población al sistema de servicios sociales y el primer nivel de intervención. Sus funciones se centran en el diagnóstico social, la elaboración de planes de intervención con la comunidad, la identificación de grupos vulnerables, la gestión del SAF, o la información, orientación y asesoramiento entre otras.

Los servicios sociales comunitarios específicos (Art. 13 de la Ley 13/2008) se centran en desarrollar y gestionar programas y centros para colectivos singulares, destacando programas de prevención de la exclusión, la atención a colectivos con déficit de autonomía o la gestión de equipamientos comunitarios, por ejemplo.

El Plan considera que Diputación cuenta con competencias y recursos para superar su rol tradicional de financiador, asumiendo nuevas funciones técnicas y relacionales como nudo central de la red de actores locales de las políticas de bienestar. Este rol se complementa con la necesidad de mejorar y estabilizar la colaboración financiera con los Ayuntamientos.

\subsection{La metodología de la elaboración del Plan. Los valores y las creencias}

Los equipos técnicos de la Diputación y del Plan diseñan una metodología para la elaboración del Plan de Acción basada en la participación como medio fundamental para la recogida e interpretación de la información.

Es necesario aludir a los valores e ideas de los actores (March y Olsen, 1997; Muller, 2002) que componen la coalición promotora del Plan y en su sistema de creencias (Sabatier, 1988; Sabatier, 1998; Sabatier y Jenkins-Smith, 1993; Sabatier y Weible, 2007) que conforman la imagen de la realidad sobre la que intervenir. Según indica Muller (2002, p. 73), «es una relación a esta imagen cognitiva que los actores organizan su percepción del sistema, van a confrontar sus soluciones y van a definir sus propuestas de acción: llama- 
GAPP. Nueva Época - N. ${ }^{\circ}$ 27, noviembre 2021 - ISSN: 1989-8991 - DOI: https://doi.org/10.24965/gapp.i27.10940 - [Págs. 85-100] ¿Cómo se define un problema público? La redefinición del problema en las políticas de bienestar locales. El caso del Plan de Acción..

remos a ese conjunto de imágenes el referencial de una política». Dentro de este referencial se encuentra el elemento multinivel como parte fundamental de todas las fases de la política, lo que lleva a la definición de la metodología participativa para la elaboración del Plan.

A continuación se muestra tabla resumen con los principales datos y herramientas utilizadas en el proceso participativo llevado a cabo.

TABla 1. Resumen de la participación en la elaboración del Plan

\begin{tabular}{|c|c|c|c|c|c|}
\hline $\begin{array}{l}\text { Técnica de } \\
\text { participación }\end{array}$ & Fechas & Número & Alcance & Tipo de participantes & Total participantes \\
\hline $\begin{array}{l}\text { Entrevistas } \\
\text { semi- } \\
\text { estructuradas }\end{array}$ & $\begin{array}{c}\text { Febrero / } \\
\text { Mayo } 2018\end{array}$ & 23 & General & $\begin{array}{l}\text { Especialistas en políticas de } \\
\text { bienestar (Universidades, } \\
\text { personas de referencia, } \\
\text { directivos Diputación...) }\end{array}$ & 23 \\
\hline $\begin{array}{l}\text { Mesas de } \\
\text { trabajo }\end{array}$ & $\begin{array}{c}\text { Marzo / } \\
\text { Abril } 2018\end{array}$ & 5 & General & $\begin{array}{l}\text { Colegios Profesionales, } \\
\text { Xunta de Galicia, FEGAMP }\end{array}$ & 16 \\
\hline $\begin{array}{l}\text { Mesas de } \\
\text { Trabajo }\end{array}$ & $\begin{array}{l}\text { Septiembre } \\
\text { / Noviembre } \\
2018\end{array}$ & 18 & Comarcal & $\begin{array}{l}\text { Personal de bienestar } \\
\text { municipal, equipos } \\
\text { entidades sociales }\end{array}$ & $\begin{array}{l}170 \text { (116 representantes } \\
\text { de entidades municipa- } \\
\text { les y } 54 \text { representantes } \\
\text { de entidades sociales) }\end{array}$ \\
\hline $\begin{array}{l}\text { Entrevistas } \\
\text { semi- } \\
\text { estructuradas }\end{array}$ & $\begin{array}{c}\text { Marzo / } \\
\text { Diciembre } 2019\end{array}$ & 86 & Local & $\begin{array}{l}\text { Personal de bienestar } \\
\text { municipal }\end{array}$ & 192 \\
\hline $\begin{array}{l}\text { Mesas de } \\
\text { Trabajo }\end{array}$ & $\begin{array}{l}\text { Noviembre } \\
2020\end{array}$ & 12 & Comarcal & $\begin{array}{l}\text { Departamentos de bienestar } \\
\text { municipales }\end{array}$ & 38 \\
\hline
\end{tabular}

Fuente: Elaboración propia a partir de datos del Plan de Acción de Servizos Sociais, Diputación de A Coruña.

En la elaboración del Plan fueron aplicadas técnicas de participación de carácter grupal e individual, convocando a un total de 439 participantes con diferentes objetivos y enfoques en su colaboración.

Este modelo de participación posibilita la interpretación de las políticas de bienestar desde una múltiple perspectiva territorial. Así, las entrevistas realizadas a personas especialistas (investigadoras universitarias, personal directivo de departamentos o equipamientos, por ejemplo) ofrecen un alcance general y permiten observar las principales dinámicas de la política y su respuesta a las situaciones socioeconómicas y demográficas del territorio.

Por su parte, las mesas de trabajo aportan una doble perspectiva. Con un alcance general se encuentran las mesas en las que participan los Colegios Profesionales de Trabajo Social, Psicología y Educación Social; la directiva de la Federación Gallega de Municipios y Provincias; y el equipo técnico de la Subdirección General de Servicios Sociales de la Consellería de Política Social. Estas mesas ofrecen información sobre la situación de las profesionales del bienestar o las relaciones entre las administraciones.

Con un alcance territorial comarcal se encuentran las 18 mesas de trabajo comarcales a las que asisten los equipos técnicos municipales y las entidades sociales financiadas por la Diputación. Su objetivo es analizar en conjunto los principales indicadores sociodemográficos del territorio, las problemáticas del trabajo cotidiano y las relaciones con la Diputación.

Las entrevistas realizadas a los equipos técnicos de 86 municipios son de naturaleza local. Las entrevistas son diseñadas como espacios de participación para analizar: (I) los recursos humanos y económicos con los que cuenta el Ayuntamiento; (II) los equipamientos de bienestar (escuelas infantiles y centros de día para personas mayores, principalmente); y (III) los programas de bienestar más destacados. Se profundiza en la realidad de los equipos, en sus problemáticas diarias y, sobre todo, en las necesidades sociales explícitas o latentes a las que no se responde.

Finalmente, se convoca una nueva ronda de mesas de trabajo comarcales a las que asisten diferentes representantes municipales para presentar las principales líneas del Plan y recoger nuevas aportaciones.

Este proceso permite observar las diferencias en los programas, enfoques metodológicos y recursos humanos y económicos a nivel local. Estas diferencias se deben a la presencia y heterogeneidad de las 
agendas locales de bienestar, que vienen determinadas por las capacidades de los actores que interactúan en la política. Son especialmente influyentes las tensiones detectadas entre los equipos municipales de bienestar, los departamentos jurídico-económicos, y los Gobiernos Locales. Estas tensiones inciden en el rendimiento institucional de la política (Subirats y Gallego, 2002, p. 18) desde la óptica local y desde la óptica de la Diputación, retomando la complejidad de la acción conjunta de Pressman y Wildawsky (1973).

Por último, se resalta que la metodología participativa contribuye al diseño del producto intermedio 3: acuerdo político-administrativo (APA) del modelo de análisis. El APA es el «conjunto estructurado de actores públicos y paraestatales que está a cargo de la implementación da política y que, por lo tanto, relacionan finalmente los postulados de la política y su aplicación concreta en un lugar determinado» (Subirats et al., 2008, p. 170).

El Plan crea un APA conformado por el equipo técnico de la Diputación y por los equipos técnicos de bienestar municipales, recogiendo la capilaridad territorial del proceso participativo, básico en la redefinición del problema y en el diseño posterior de las nuevas medidas de la política.

\subsection{Los documentos integrantes del Plan}

El Plan de Acción de Servicios Sociales se compone de: (I) dieciocho diagnósticos comarcales sobre la situación de las políticas de bienestar a nivel comarcal y el estado de los departamentos municipales de bienestar; (II) un diagnóstico provincial sobre la situación de las políticas de bienestar a nivel provincial y los servicios de la Diputación; (III) un Marco Estratégico para la interpretación de la situación actual y las principales líneas de desarrollo de la política; y (IV) un Plan de Acción con las principales recomendaciones y medidas.

El conjunto se completa con una Galería de Experiencias Comparadas en donde se muestran programas de interés liderados por Ayuntamientos de menor población, y con un Buscador de Equipamientos de Bienestar con información sobre los centros operativos en la provincia.

Los documentos muestran, en términos locales y comarcales, los principales indicadores socioeconómicos y demográficos, así como la situación de los servicios de bienestar municipales y sus principales programas. A nivel provincial se analiza la actividad de la Diputación que, por un lado, cuenta con servicios de bienestar propios (el programa de Teleasistencia y la gestión de varios equipamientos) y, por otro lado, establece una relación con los Ayuntamientos de menos de 20.000 habitantes basada en la financiación de equipos administrativos y técnicos, y de horas de SAF de libre concurrencia.

Una vez analizada la realidad social y departamental, se establecen conclusiones y recomendaciones a diferentes niveles territoriales atendiendo al Marco Estratégico elaborado. Además, de modo coherente con estas recomendaciones, en el documento de Plan de Acción se recogen las medidas específicas diseñadas para el Departamento de Servicios Sociales de la Diputación.

\subsubsection{El Marco Estratégico}

El Marco Estratégico se considera central en el conjunto de los documentos del Plan de Acción, ya que su objetivo es la:

... fijación de un marco conceptual para la interpretación de la situación de partida de nuestra sociedad y del modelo de política de bienestar que se implementa desde los Ayuntamientos y desde la Diputación para, a partir de su análisis, establecer un nuevo modelo de política ${ }^{7}$. (Diputación de A Coruña, 2020a, p. 10).

El Marco Estratégico,

... expone los elementos centrales que deberían guiar la refocalización de las políticas de bienestar, define (...) la realidad de la prestación de servicios de bienestar, traza las principales características de la propuesta del nuevo modelo de política multinivel a liderar desde el Área de Bienestar de la Diputación y (...) diseña las líneas básicas de este nuevo modelo ${ }^{8}$. (Diputación de A Coruña, 2020a, p. 11).

\footnotetext{
7 Original en gallego, traducción del autor.

8 Original en gallego, traducción del autor.
} 
GAPP. Nueva Época - N. ${ }^{\circ}$ 27, noviembre 2021 - ISSN: 1989-8991 - DOI: https://doi.org/10.24965/gapp.i27.10940 - [Págs. 85-100] ¿Cómo se define un problema público? La redefinición del problema en las políticas de bienestar locales. El caso del Plan de Acción..

El Marco sienta los valores que guían la nueva política que son los de compromiso, solidaridad, integración, participación, proximidad, liderazgo público, inteligencia y diversidad (Diputación de A Coruña, 2020a, pp. 53-55). Además, se exponen las bases conceptuales sobre las que se asienta el nuevo modelo (Adelantado et al., 2000; Brugué, 2017; Brugué y Gomà, 1998; Del Pino y Rubio Lara, 2016).

En el documento se estudia el contexto demográfico y socioeconómico sobre el que actúan las políticas de bienestar, que se caracteriza por: (I) el retroceso demográfico general; (II) el surgimiento de la soledad como fenómeno social; (III) la expansión de la desigualdad y vulnerabilidad social; y (IV) la familiarización de los cuidados. (Diputación de A Coruña, 2020a, pp. 20-37).

Como respuesta a estos factores, en el Marco estratégico se define un nuevo modelo de política cuyo resumen se observa en la siguiente imagen.

\section{Figura 2. Resumen del Marco Estratégico del Plan de Acción de Servicios Sociales}

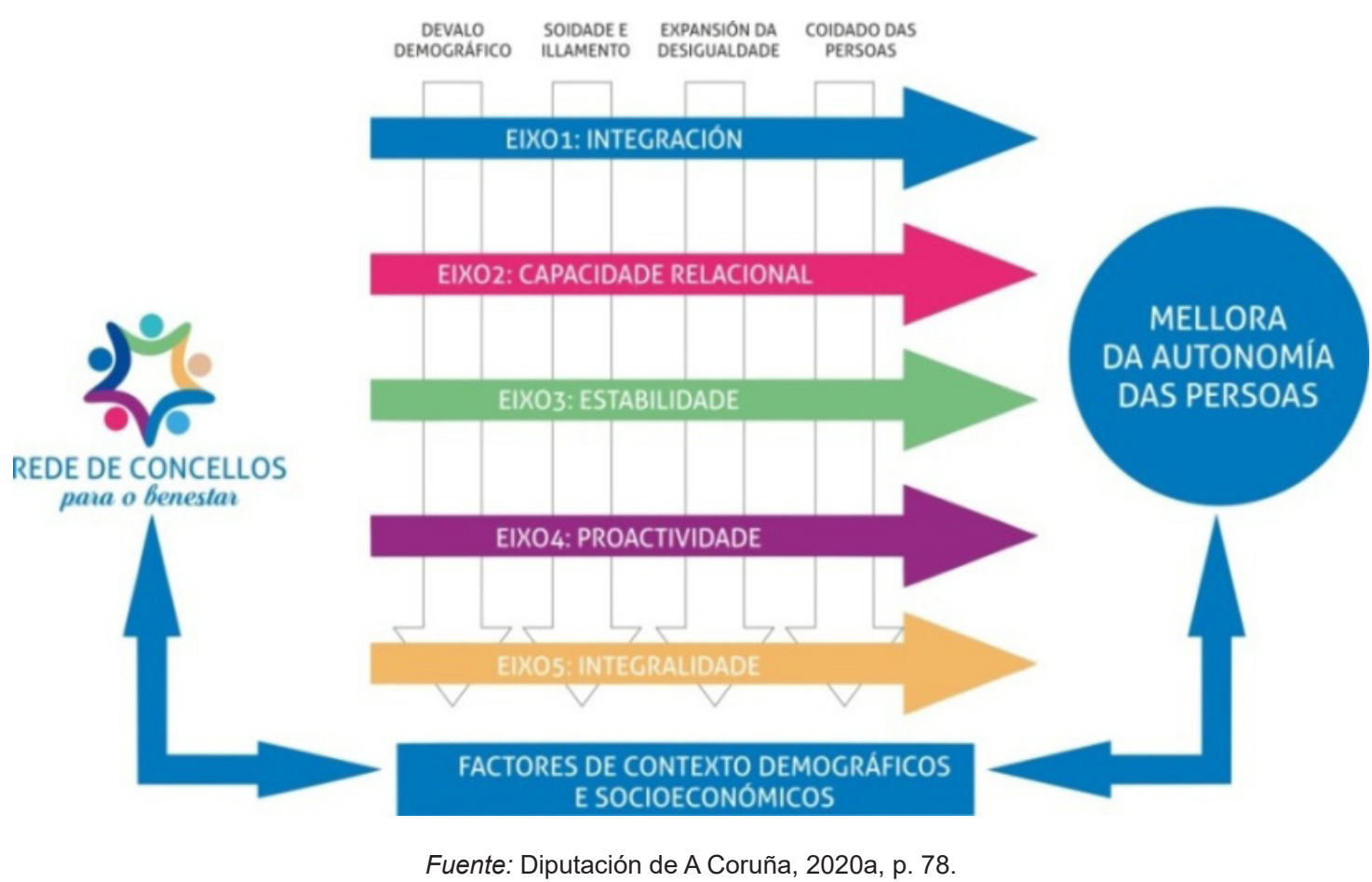

La nueva política tiene el objetivo de mejorar de la autonomía de las personas a través de la intervención en los factores del contexto. Esta intervención se lleva a cabo desde la Rede de Concellos para o Benestar, conformada por los Ayuntamientos menores de 20.000 habitantes de la provincia, por el Área de Bienestar de la Diputación y por las entidades del Tercer Sector de Acción Social.

El nuevo modelo de política es (I) integrado, (II) relacional, (III) estable, (IV) proactivo, e (V) integral; y sus las líneas generales se avanzan en el Marco Estratégico (Diputación de A Coruña, 2020a, pp. 64-77), pero su desarrollo y las medidas específicas se detallan en el documento de Plan de Acción (Diputación de A Coruña, 2020c).

El Marco Estratégico revisa las reglas institucionales del modelo anterior de política, redefiniendo el problema público (producto intermedio 1 del modelo de análisis), el programa de actuación político-administrativo (producto intermedio 2), y el acuerdo político-administrativo (producto intermedio 3). Además, el Marco sienta las bases para la implementación (etapa 3) y evaluación (etapa 4) de la política.

\subsubsection{Los Diagnósticos. Las agendas locales del bienestar}

En los documentos que integran el Plan destaca la presencia de dieciocho Diagnósticos Comarcales y un Diagnóstico Provincial, que muestran las variables demográficas y socioeconómicas que marcan las políticas de bienestar. 
GAPP. Nueva Época - N. ${ }^{\circ}$ 27, noviembre 2021 - ISSN: 1989-8991 - DOI: https://doi.org/10.24965/gapp.i27.10940 - [Págs. 85-100] ¿Cómo se define un problema público? La redefinición del problema en las políticas de bienestar locales. El caso del Plan de Acción..

En los diagnósticos se analiza la dotación de equipamientos en el territorio y la actividad de los Ayuntamientos y de la Diputación, incluyendo aspectos como los recursos humanos o los presupuestos. Además de la recopilación de datos, los diagnósticos recogen las valoraciones realizadas por los equipos técnicos sobre la actividad de los departamentos y las necesidades sociales que en la actualidad no se ven atendidas (o sobre las que se podría mejorar su atención).

Una de las partes destacadas es la relacionada con los equipamientos de bienestar y, concretamente, con la evolución de las plazas de los centros de bienestar para las personas mayores (residenciales y no residenciales o centros de día), actualizando la metodología del Mapa galego de equipamentos e servizos sociais de la Vicepresidencia da Igualdade e do Benestar de la Xunta de Galicia (2008) ${ }^{9}$. Este análisis cuantifica el (I) número de plazas actual y el número objetivo de los equipamientos en función de la ratio de referencia ${ }^{10}$; (II) el porcentaje actual de cobertura de los equipamientos; y (III) las plazas que serían necesarias para alcanzar los objetivos establecidos. Es decir, se cuantifica la infradotación referida en múltiples ocasiones por los equipos técnicos en la elaboración del Plan.

En relación a los centros de día para la atención a las personas mayores (considerados de elevado impacto en relación a la prevención, a los cuidados, a la conciliación y al empleo de proximidad), se obtiene un déficit 2.156 plazas en la provincia, destacando la ausencia de este servicio en comarcas como Fisterra, por ejemplo (Diputación de A Coruña, 2020b, p. 114).

En los diagnósticos también son analizados los presupuestos municipales inicialmente aprobados para el año 2018 y el programa de servicios sociales y promoción social representado por la política de gasto $23^{11}$ de la estructura presupuestaria. La inversión municipal por habitante se considera heterogénea, oscilando entre el $33 \%$ de peso de la política de gasto 23 sobre el total del presupuesto del Ayuntamiento de Boimorto (con más de $433 € /$ habitante), y cifras inferiores al $5 \%$ en Ayuntamientos como Val do Dubra (con $24 € /$ habitante) o Fisterra ( $37 € /$ habitante). El promedio del peso asciende al $13 \%$, en tanto que la inversión ronda los $120 € /$ habitante, detectando problemas en la confiabilidad del dato que incrementan la complejidad de la interpretación.

Finalmente, los equipos municipales de bienestar reconocen las carencias y limitaciones provocadas por el uso de la vía incentivadora por parte de la Diputación como principal herramienta de relación con los Ayuntamientos. Esta percepción se basa en su inestabilidad derivada de la temporalidad anual y en su exclusividad, ya que se considera limitado y parcial el apoyo metodológico recibido de la Diputación.

En el primero de los aspectos, es destacable la relación existente entre la temporalidad de la financiación y las dificultades jurídicas a la hora de contratar personal (tanto técnico como de apoyo administrativo) a nivel municipal, lo que provoca disfunciones y discontinuidades en la política pública. De todos modos, la presencia de estas dificultades es heterogénea, lo cual supone la existencia de márgenes en la interpretación de la normativa por parte de actores jurídicos locales con rol de gatekeepers o habilitadores (Dente y Subirats, 2014; Font, 1999) que varían el rendimiento de la política.

Con respecto al segundo elemento, la utilización intensiva de las convocatorias anuales de financiación provoca la focalización de la actividad del departamento provincial de bienestar en la gestión administrativa del ciclo de la subvención. Este hecho, y la senda de dependencia que genera, limitan la detección y respuesta a las necesidades técnicas y metodológicas de los equipos locales.

Los diagnósticos ratifican que la amplitud y profundidad del proceso participativo son elementos cruciales en la redefinición del problema público, ampliando su perímetro e identificando nuevos campos sobre los que intervenir.

9 El Mapa gallego de servicios sociales es el instrumento de planificación que define las áreas sociales de y los criterios de dotación de centros y servicios sociales (Art.44.2 de la Ley 13/2008 de servicios sociales de Galicia).

10 En el Mapa se analizan los índices de cobertura para las plazas no residenciales y residenciales para la atención a mayores de 65 años, tomando el $5 \%$ para las residenciales y el $2 \%$ para las no residenciales. Esta metodología se actualiza y comarcaliza en Diputación da Coruña (2020b, pp. 113-114).

11 El Anexo I de la Orden EHA/3565/2008 define la política de gasto 23: Servicios sociales y promoción social: «Se imputarán a esta política de gasto los llevados a cabo por la entidad local para la promoción de la igualdad de género, promoción y reinserción social de marginados, así como para la gestión de los servicios sociales; prestación de servicios a personas dependientes y de asistencia social, residencias de ancianos y otros de naturaleza análoga. También se incluirán las transferencias finalistas a entidades y familias que colaboren en la consecución de estos fines. Se incluirán los gastos correspondientes a la Administración General, que corresponderán a las actividades dirigidas a la planificación, coordinación, control, organización, gestión administrativa y desarrollo de funciones de apoyo de los distintos centros directivos que integran la política de gasto». 
GAPP. Nueva Época - N. ${ }^{\circ}$ 27, noviembre 2021 - ISSN: 1989-8991 - DOI: https://doi.org/10.24965/gapp.i27.10940 - [Págs. 85-100] ¿Cómo se define un problema público? La redefinición del problema en las políticas de bienestar locales. El caso del Plan de Acción..

Manuel Joaquín Pérez Castreje

\subsubsection{El Plan de Acción}

El documento de Plan de Acción (Diputación de A Coruña, 2020c) recoge las líneas de actuación y medidas que representan el nuevo modelo de política de bienestar. Contiene cuarenta y siete actuaciones estructuradas en cinco ejes estratégicos a implementar en seis anualidades. Además, incluye una primera definición de un sistema de seguimiento, control y evaluación de la implementación del Plan en el que se integran los equipos locales de bienestar.

El Plan de Acción contempla actuaciones a implementar desde la Diputación, sentando las bases institucionales para el nuevo modelo de colaboración con los municipios y, a partir de ahí, avanzar en el diseño e implementación de medidas sustantivas.

Las actuaciones incluyen medidas como (I) el fortalecimiento del departamento de bienestar mediante la creación de un Área propia, incrementando sus recursos a nivel central y territorial; (II) la conformación de un Centro de Recursos para el apoyo técnico a los equipos municipales; (III) el establecimiento de relaciones estables entre Diputación, Ayuntamientos y los actores sociales del territorio; (IV) la conformación de un nuevo modelo de financiación de los departamentos municipales y de las entidades sociales; o (V) la mejora de programas propios de la Diputación, por ejemplo (Diputación de A Coruña, 2020c).

El Plan de Acción establece las medidas para superar la centralidad de la vía incentivadora, avanzando en la prestación de servicios técnicos, metodológicos y jurídicos por parte de la Diputación.

\subsubsection{Las limitaciones del Plan de Acción}

El estudio del Plan de Acción permite observar la complejidad ${ }^{12}$ de una política y la dificultad de su ajuste a los modelos analíticos. Así, el contenido del Plan presenta matices con respecto al contenido del producto intermedio 2: programa de actuación político-administrativo (PPA) del modelo de análisis de referencia.

El PPA, según Subirats et al. (2008, pp. 151-152), «incorpora el conjunto de normas y actos reglamentarios que los parlamentos, los gobiernos y los organismos o autoridades a cargo de la implementación consideran necesarias para la aplicación de una política pública». A nivel formal, el PPA incorpora elementos como decretos actos administrativos, reglamentos o directrices emitidas por las administraciones competentes. A nivel material integra elementos como los objetivos normativos de la solución prevista, la definición de los grupos objetivo o los sistemas de organización administrativa.

Los documentos que integran el Plan de Acción de Servicios Sociales responden a una visión parcial del PPA, centrándose en los elementos materiales de la política en detrimento de los elementos formales y jurídicos. Así, a pesar de que se apuntan los elementos clave de la política, no se cierran las herramientas reglamentarias necesarias para su desarrollo, por lo que se está ante un PPA parcial o incompleto que deberá ser objeto de desarrollo a lo largo de la implementación del Plan.

Además, si bien en el modelo de análisis el PPA debe integrar los elementos evaluativos de la política pública, en el Plan de Acción estos se apuntan de modo inicial sin profundizar en su desarrollo. A pesar de todo, se fijan sus bases participativas y territorializadas, con lo que esto supone en el proceso de retroalimentación de la política (Diputación de A Coruña, 2020c, pp. 51-54).

\section{LA EVOLUCIÓN DE LA DEFINICIÓN DEL PROBLEMA PÚBLICO Y SUS EFECTOS EN LA POLÍTICA}

El Plan de Acción cuenta con una coalición promotora constituida por el equipo de la Diputada Delegada de Igualdade e Benestar en la legislatura 2015-2019 y los equipos técnicos de bienestar de la Diputación y del Plan. Estos actores lideran la redefinición del problema al que debe responder el nuevo modelo de la política.

La coalición promotora integra la complejidad de la política y el elemento multinivel en su conjunto de imágenes o creencias (Muller, 2002; Sabatier, 1988; Sabatier, 1998; Sabatier y Jenkins-Smith, 1993; Sabatier y Weible, 2007), lo que da entrada a los equipos técnicos de bienestar municipales como actores y, por lo tanto, varía el enfoque en la definición del problema. Esta integración supone la ampliación del perímetro del problema público, superando la definición previa centrada únicamente en la infradotación financiera local a la que da respuesta la Diputación mediante las convocatorias anuales de subvenciones.

\footnotetext{
12 Dunn (1994) se refiere los conceptos «tangled» $\mathrm{y}$ «disjointed».
} 
GAPP. Nueva Época - N. ${ }^{2}$ 27, noviembre 2021 - ISSN: 1989-8991 - DOI: https://doi.org/10.24965/gapp.i27.10940 - [Págs. 85-100] ¿Cómo se define un problema público? La redefinición del problema en las políticas de bienestar locales. El caso del Plan de Acción..

A lo largo del Plan de Acción se establece una caracterización de la anterior política pública marcada por: (I) la fragmentación; por (II) la atomización y la dificultad para la creación de redes de actores locales; por (III) la discontinuidad, el corto plazo y la intensidad de las tareas de gestión administrativa; por (IV) la reactividad; por (V) las limitaciones en los equipos; y finalmente $(\mathrm{VI})$ por la debilidad institucional del Área de Bienestar de la Diputación (Diputación de A Coruña, 2020a).

La fragmentación como característica de la política, observada desde una perspectiva supralocal, supone la existencia de diferencias en el abanico e intensidad de los servicios de bienestar locales que no responden a los contextos sociales. Esta situación genera una inequidad final para la ciudadanía que no es paliada a través de la colaboración con las entidades sociales locales, ya que el trabajo conjunto es escaso. Las políticas de bienestar locales se caracterizan por ser atomizadas y, en términos generales, no generar sinergias.

Además, la combinación entre la limitación de los recursos locales y el modelo de financiación anual genera dependencia municipal, ya que el modelo de la subvención anual, según Gallego et al. (2003, p. 167):

... se está convirtiendo en un instrumento a través del cual los poderes públicos transforman los derechos de los ciudadanos en una simple oferta contingente y discrecional, ya que las administraciones públicas pueden establecer y modificar los criterios y requisitos o incluso suprimir las subvenciones, sin que asistan a los beneficiarios posibles derechos adquiridos.

Esta situación, sumada al incremento de las tareas de carácter administrativo a asumir por los departamentos municipales, conlleva la presencia de rupturas en los programas a causa de la limitación (en número, en estabilidad y en perfiles profesionales) de los equipos, existiendo una tendencia a la reactividad como premisa de actuación frente a las necesidades sociales. La reactividad genera una senda de dependencia asentada en las rutinas de los equipos municipales, que deriva en la hiperfocalización en los programas activos (sobre todo SAF) y obvia la emergencia e inclusión en la agenda de nuevas necesidades sociales (Diputación de A Coruña, 2020a, p. 45).

Por otra parte, la senda de dependencia presente en el departamento provincial de bienestar determina su organización interna e influye en las características del modelo de política. La hiperfocalización en el seguimiento administrativo del ciclo de las subvenciones supone la concentración de la atención organizacional (March y Olsen, 1997), y limita las capacidades técnicas para la definición de modelos sustantivos de políticas de bienestar; generando nuevas necesidades administrativas que profundizan en el modelo vigente (Diputación de A Coruña, 2020a, pp. 50-52).

Frente a esta situación, el Plan de Acción redefine el problema público atendiendo a la ausencia de un modelo estable de política de bienestar local que responda a las necesidades técnicas y económicas municipales. Así, la Diputación, en el marco de sus competencias, puede prestar apoyo a los Ayuntamientos a través de un nuevo modelo de política de bienestar local.

El Plan de Acción propone avanzar en un modelo de política (I) integrado; (II) relacional; (III) estable; (IV) proactivo; e (V) integral. En el modelo, el departamento de bienestar de la Diputación es el nudo central de la Red (Diputación de A Coruña, 2020a, pp. 53-67).

Este modelo incluye un PPA (producto intermedio 2) que propone: (I) la redefinición del departamento de bienestar de la Diputación, encargado de liderar y coordinar la política pública; (II) la definición de los principales grupos objetivo de la política, focalizados en el propio departamento de bienestar, en los Ayuntamientos y en las entidades sociales; y (III) un nuevo enfoque y objetivos de la política.

EI PPA focaliza las actividades en los Ayuntamientos y en el departamento de bienestar de la Diputación como grupos objetivo, introduciendo unas nuevas reglas institucionales en su relación y estableciendo canales con entidades sociales y otras instituciones para la generación de nuevas metodologías de intervención.

EI APA (producto intermedio 3) de la política pública estaría compuesto inicialmente por el departamento de bienestar de la Diputación y por los equipos técnicos de bienestarlocales, conformando la Rede de Concellos para o Benestar, a la que se añadirían las entidades sociales financiadas por la Diputación.

La constitución de la Red responde a la atomización detectada y supone una modificación sustancial del modelo centrado en la Diputación como ente financiador, en el que los Ayuntamientos o entidades sociales contaban con un rol reactivo. Las nuevas reglas se centran en la estabilidad y en la reducción de la asimetría como elementos opuestos a la inestabilidad anual y a la asimetría de la vía incentivadora.

Así, la profundidad de metodología utilizada en la elaboración del Plan contribuye a: (I) integrar la complejidad multinivel como parte de la política; (II) determinar la nueva definición y el perímetro del problema público; y (III) conformar un APA para el nuevo modelo de política bajo el modelo de Red. 


\section{CONCLUSIONES Y APORTACIONES DE LA INVESTIGACIÓN}

A lo largo del artículo se estudia la redefinición del problema público relacionado con las políticas locales de bienestar llevado a cabo en la realización del Plan de Acción de Servicios Sociales de la Diputación de A Coruña.

El modelo previo de la política asumía una definición del problema público centrada en la infradotación de recursos financieros de los Ayuntamientos para sostener las políticas de bienestar locales. Esta situación era paliada, en parte, por una política basada en la convocatoria de diferentes líneas de financiación anual por parte de la Diputación.

La utilización de las subvenciones como principal herramienta de relación entre administraciones genera unas reglas institucionales y una lógica de lo apropiado (March y Olsen, 1997) que deriva en una senda de dependencia marca la actuación del departamento de bienestar provincial, la percepción de sus necesidades y su atención e inteligencia organizacional. Además, el modelo influye en la actuación de los departamentos de bienestar municipales que captan recursos a través del ciclo de la subvención, generando y asentando rutinas que van en detrimento de la percepción y respuesta a los problemas sociales locales.

La distancia entre financiador (Diputación) y ente prestador de servicios (Ayuntamientos), y la hiperfocalización del trabajo operativo del financiador en el seguimiento administrativo de la subvención genera limitaciones en los efectos de la financiación y profundiza en la fragmentación como característica de la política.

La fragmentación se ve reforzada ya que el equipo de la Diputación no genera enfoques metodológicos comunes para los programas de bienestar locales, por lo que no hay un modelo de política local común. Esta ausencia se debe a la tensión operativa creada por la utilización de la subvención como herramienta de relación entre las administraciones, que focaliza su atención en su ciclo administrativo en detrimento de la detección/respuesta a otras necesidades de los Ayuntamientos. Se conforma una lógica de lo apropiado firme y estable en la política pública.

La utilización de las subvenciones como herramienta fundamental de una política multinivel supone su institucionalización al generar normas, reglas, valores, estructuras, rutinas, sistemas de creencias, conceptos compartidos, elementos culturales y cognitivos, recursos... (March y Olsen, 1997; Muller, 2002; Ostrom, 2013; Parrado, 2015; Peters, 2003; Sabatier, 1998; Salvador, 2003). La herramienta moldea la política a través de la adaptación de los enfoques y valores de los actores a su ciclo administrativo, definiendo necesidades y limitando las alternativas de elección. De hecho, a lo largo de la elaboración del Plan de Acción se verifica que es más sencillo para los actores internos de la administración financiadora conseguir sustanciales incrementos presupuestarios ${ }^{13}$ que variar la herramienta.

La tradición de la utilización de las convocatorias de subvenciones genera unas reglas asimétricas (Pérez, 2020) y circulares (Medir, 2015) que no responden a necesidades municipales diferentes de la financiera, y cuya reproducción afianza y consolida el modelo. La subvención se convierte en institución.

Frente a esta realidad, la redefinición del problema no responde a la presencia de evaluaciones del anterior modelo de política (Pérez, 2020, p. 239), sino a la presencia de una coalición promotora con un referencial de la política (Gomà y Subirats, 1998; Muller, 2002; Sabatier, 1988; Sabatier, 1998; Sabatier y Jenkins-Smith, 1993; Sabatier y Weible, 2007) diferente al previo, que aprovecha una ventana de oportunidad (Kingdon, 1984) para movilizar los recursos suficientes y promover una nueva política pública.

La presencia e importancia de los valores relacionados con la complejidad y el elemento multinivel en los sistemas de creencias de la coalición lleva a la integración de los equipos técnicos de bienestar municipales como actores en la redefinición del problema, a diferencia de lo sucedido en el anterior modelo. Esto amplía y profundiza el perímetro del problema, suponiendo el reconocimiento de la «máxima confluencia multinivel» (Gomà y Subirats, 1998, pp. 392-394) como característica de las políticas de bienestar.

La redefinición del problema público (Diputación de A Coruña, 2020a), centrada en la ausencia de un modelo estable de política de bienestar que responda a las necesidades técnicas y económicas municipales, supone un nuevo enfoque. El Plan de Acción avanza en la creación de un modelo de bienestar local adaptable al territorio, abriendo campos de actuación desde lo sustantivo, y diseñando unas nuevas reglas institucionales basadas en la red.

En la elaboración del Plan se observa la presencia y heterogeneidad de las agendas locales del bienestar como elemento que explica la presencia o ausencia de diferentes programas en respuesta a las pro-

13 Ver nota 5. 
GAPP. Nueva Época - N. ${ }^{\circ}$ 27, noviembre 2021 - ISSN: 1989-8991 - DOI: https://doi.org/10.24965/gapp.i27.10940 - [Págs. 85-100] ¿Cómo se define un problema público? La redefinición del problema en las políticas de bienestar locales. El caso del Plan de Acción..

Manuel Joaquín Pérez Castreje

blemáticas sociales. Esta heterogeneidad se plasma en sustanciales diferencias de inversión por habitante, la variedad de modelos de gestión del SAF como principal programa local, o la presencia/ausencia de programas específicos que incidan en necesidades emergentes, como pueden la soledad, la prevención de adicciones en la adolescencia o los cuidados a personas cuidadoras.

La imposibilidad de vincular la relación de causalidad entre las agendas y aspectos como el grado de ruralidad, el nivel de envejecimiento de la población, o los actores políticosde los Gobiernos locales afirma la vigencia del modelo del garbage can o bote de basura (March y Olsen, 1997) y su capacidad explicativa para la conformación de las agendas (Aguilar, 2000, pp. 31-33; Cobb y Elder, 1984, p. 115) a raíz de la confluencia de problemas, soluciones, actores y oportunidades de elección.

En este esquema destaca el triángulo básico de actores que integran las políticas de bienestar locales. Este se conforma por el Gobierno Local, por los equipos técnicos de bienestar, y por los equipos jurídicofinancieros (Boldú et al., 2020, pp. 133-134), destacando especialmente el rol de estos últimos como gatekeepers o habilitadores (Dente y Subirats, 2014; Font, 1999).

La diferente capacidad de movilización de recursos (Subirats et al., 2008) de los actores integrantes del triángulo mencionado, y el tipo de interacción (Font, 1999), determinan la agenda local del bienestar. Esta situación explica las diferencias entre Ayuntamientos de características semejantes y la imposibilidad de identificar un modelo homogéneo de política local de bienestar desde una óptica supramunicipal.

Frente a esta situación, el Plan de Acción pretende avanzar en la creación y consolidación de un modelo homogéneo y adaptable al territorio que responda a la presencia de situaciones sociales que no habían sido tratadas o se trataban limitadamente.

De todos modos, no se identifican aspectos de carácter formal o propuestas de regulación en losdocumentos que integran el Plan de Acción. Esta limitación generala necesidad de nuevos espaciosen los que se definan estos elementos. En estos espacios (internos y de limitada visibilidad) se integrarán actores que inicialmente no han formado parte del APA ni de los actores que redefinieron el problema público, como es el caso de los técnicos jurídicos. Estos, ejerciendo el rol de habilitadores (Dente y Subirats, 2014; Subirats et al., 2008), plasmarán su influencia en los textos regulatorios que desarrollen la política, interactuando con la coalición promotora e introduciendo sus propias imágenes (Muller, 2002) y sistemas de creencias (Sabatier, 1988; Sabatier, 1998; Sabatier y Jenkins-Smith, 1993; Sabatier y Weible, 2007).

Con el resultado de estas nuevas interacciones se estaría ante la posibilidad de una nueva definición del problema público (total o parcial) atendiendo a los valores, imágenes, creencias o intereses de los nuevos actores incorporados (Stone, 1989). Esto supone la discusión de facto de la centralidad de la coalición promotora inicial y posibilita la revisión de la política, entrando en un nuevo ciclo incluso antes de la generación de efectos a corto y medio plazo.

\section{REFERENCIAS BIBLIOGRÁFICAS}

Adelantado, J. (2011). La dimensió simbòlica en les polítiques autonòmiques de serveis socials i assistència social. En R. Gallego, y J. Subirats (eds.), Autonomies i desigualtats en Espanya: percepcions, evolució social i politiques de benestar (pp. 319-340). Institut d’Estudies Autonòmics.

Adelantado, J., Noguera, J. A. y Rambla, X. (2000). El marco de análisis: las relaciones complejas entre estructura social y políticas sociales. En Adelantado, J. (ed.), Cambios en el Estado de Bienestar. Políticas sociales y desigualdades en España (pp. 23-31). Icaria-UAB.

Aguilar Villanueva, L. F. (2000). Problemas públicos y agenda de gobierno. Miguel Ángel Porrúa.

Ayala Espino, J. (1999). Instituciones y economía: una introducción al neoinstitucionalismo económico. Fondo de Cultura Económica.

Bardach, E. (1998). Los ocho pasos para el análisis de políticas públicas. CIDE-Miguel Angel Porrua.

Boldú, M., Casademont, X. y Ginesta, M. (2020). Qui goberna els serveis socials en el món local. Colegio Oficial de Trabajo Social de Cataluña.

Brugué, J. (2017). Administració i Govern: accionssense política pública. En R. Gomà y J. Subirats (coords.), Canvid'època i de polítiques públiques a Catalunya (pp. 36-41). Critic-Sccl.

Brugué, J. y Gomà, R. (coords.) (1998). Gobiernoslocales y políticas públicas. Bienestar social, promoción económica y territorio. Ariel.

Brugué, J., Garola, A., Muñoz, F., Ulied, A., Ximeno, A. y Xalabarder, M. (2014). ¿Qué estrategias para qué retos? 25 criterios para el diseño de estrategias en el desarrollo local. Diputación de Barcelona.

Cobb, R. y Elder, C. (1984). Formación de la agenda. El caso de la política de los ancianos. En L. F. Aguilar Villanueva (ed.), Problemas públicos y agenda de gobierno (pp. 77-104). Miguel Ángel Porrúa. 
GAPP. Nueva Época - N. ${ }^{\circ}$ 27, noviembre 2021 - ISSN: 1989-8991 - DOI: https://doi.org/10.24965/gapp.i27.10940 - [Págs. 85-100] ¿Cómo se define un problema público? La redefinición del problema en las políticas de bienestar locales. El caso del Plan de Acción..

Manuel Joaquín Pérez Castreje

Del Pino, E. y Rubio Lara, M. J. (eds.) (2016). Los estados del bienestar en la encrucijada. Políticas sociales en perspectiva comparada. Tecnos.

Dente, B. y Subirats, J. (2014). Decisiones públicas. Análisis y estudio de los procesos de decisión en políticas públicas. Ariel.

Diputación de A Coruña (2020a). Marco Estratéxico do Plan de Acción de Servizos Sociais. https://www.dacoruna.gal/ files/1216/3360/8137/Marco_Estratexico.pdf

Diputación de A Coruña (2020b). Os servizos sociais na provincia da Coruña. Plan de Acción de Servizos Sociais. https://www.dacoruna.gal/files/3216/3360/8100/os_servizos_sociais_na_provincia_da_Coruna.pdf

Diputación de A Coruña (2020c). Plan de Acción de Servizos Sociais. https://www.dacoruna.gal/files/1116/3360/8146/ Plan_de_accion_Servizos_Sociais.pdf

Dunn, W. (1994). Public policy analysis. Anintroduction. Prentice-Hall.

Dye, T. (1992). Understanding public policy. Prentice-Hall.

Ebbinghaus, B. (2005). Can Path Dependence Explain Institutional Change? Two Approaches Applied to Welfare State Reform (Discussion Paper, 05/2). Max-Planck-Institut für Gesellschaftsforschung. https://www.mpifg.de/pu/mpifg_ dp/dp05-2.pdf

Font, N. (1999). Estudios de caso: objeto y técnicas de análisis. En S. Aguilar, N. Font y J. Subirats (eds.), Política ambiental en España. Subsidiariedad y Desarrollo Sostenible (pp. 99-112). Tirant lo Blanch.

Gallego, R., Gomà, R. y Subirats, J. (2003). Estado de bienestar y comunidades autónomas. Tecnos UPF.

Gomà, R. y Subirats, J. (1998). Políticas públicas en España. Contenidos, redes de actores y niveles de gobierno. Ariel.

Hall, P. A. y Taylor, R. C. R. (1996). Political Science and the Three New Institutionalism. Political Studies, XLIV, 936957. https://doi.org/10.1111/j.1467-9248.1996.tb00343.x

Jones, Ch. O. (1970). An Introduction to the Study of Public Policy. Duxbury Press.

Kingdon, J. (1984). Agendas, Alternatives and Public Policies (nueva edición ampliada en 1995). Little Brown.

Lakoff, G. (2007). No pienses en un elefante. Lenguaje y debate político. Editorial Complutense, S. A. (Foro Complutense).

Laswell, H. (1951). La orientación hacia las políticas. En L. F. Aguilar Villanueva (ed.), El estudio de las políticas públicas (pp. 79-104). Miguel Ángel Porrúa.

Laswell, H. (1971). La concepción emergente de las ciencias de políticas. En L. F. Aguilar Villanueva (ed.), El estudio de las políticas públicas (pp. 105-118). Miguel Ángel Porrúa.

Lindblom, C. (1991). El proceso de elaboración de políticas públicas. Ministerio para las Administraciones Públicas.

March, J. y Olsen, J. (1997). El redescubrimiento de las instituciones. Fondo de Cultura Económica.

Medir Tejado, LI. (2015). Los convenios de colaboración en materia educativa entre la Generalitat de Catalunya y los gobiernoslocales: morfología y contenidos (1999-2010). Revista catalana de dret públic, 50, 161-183. http:// revistes.eapc.gencat.cat/index.php/rcdp/article/view/10.2436-20.8030.01.49

Meny, I. y Thoenig, J. C. (1992). Las políticas públicas. Ariel.

Metcalfe, L. (1993). Gestión pública: de la imitación a la innovación. En J. Brugué y J. Subirats (coords.), Lecturas de Gestión Pública (pp. 77-100). Ministerio de Administraciones Públicas.

Muller, P. (2002). Las Políticas Públicas (1. ${ }^{a}$ ed.). Universidad Externado de Colombia.

North, D. (1990). Institutions, Institutional Change and Economic Performance. Cambridge University Press.

Ostrom, E. (2013). Comprender la diversidad institucional. KRL Ediciones.

Parrado, S. (2015). El análisis de la Gestión Pública. Tirant lo Blanch.

Parsons, W. (1995). Public Policy, An Introduction to the Theory and Practice of Policy Analysis. Edward Elgar.

Pérez Castreje, M. J. (2020). El nacimiento de una institución. El diseño, creación y evolución del Consorcio Galego de Servizos de lgualdade e Benestar. INAP.

Peters, G. (2003). El nuevo institucionalismo. La teoría institucional en Ciencia Política. Gedisa.

Pressman, J. L. y Wildavsky, A. (1973). Implementation. Berkeley University Press.

Sabatier, P. A. (1988). An Advocacy Coalition Model of Policy Change and the Role of Policy Orientated Learning Therein. Policy Sciences, 21, 129-168. https://link.springer.com/article/10.1007\%2FBF00136406

Sabatier, P. A. (1998). The advocacy coalition framework:revisionsand relevance for Europe. Journal of European Public Policy, 5(1), 98-130, http://doi.org/10.1080/13501768880000051

Sabatier, P. A. y Jenkins-Smith, H. (eds.) (1993). Policy Change and Learning: An Advocacy Coalition Approach. Westview Press.

Sabatier, P. A. y Weible, Ch. M. (2007). A Guide to the Advocacy Coalition Framework. En F. Fischer, G. Miller y M. Sidney (eds.), Handbook of Public Policy Analysis. Theory, Politics and Methods (pp. 123-136). CRC Press.

Salvador, M. (2003). Instituciones y políticas públicas en la gestión de los recursos humanos de las comunidades autónomas [Tesis doctoral]. Universitat Pompeu Fabra, Departamento de Ciencias Políticas y Sociales. http://hdl. handle.net/10803/7249

Stone, D. A. (1989). Casual stories and the formation of policy agendas. Political Science Querterly, 104(2), 281-300. https://doi.org/10.2307/2151585

Subirats, J. y Gallego, R. (eds.) (2002). Veinte años de autonomías en España: leyes, políticas púbicas, instituciones y opinión pública. CIS. 
GAPP. Nueva Época - N. ${ }^{\circ}$ 27, noviembre 2021 - ISSN: 1989-8991 - DOI: https://doi.org/10.24965/gapp.i27.10940 - [Págs. 85-100]

¿Cómo se define un problema público? La redefinición del problema en las políticas de bienestar locales. El caso del Plan de Acción..

Manuel Joaquín Pérez Castreje

Subirats, J., Knoepfel, P., Larrue, C. y Varone, F. (2008). Análisis y gestión de políticas públicas. Ariel.

Tolbert, P. S. y Zucker, L. G. (1996). The Institutionalization of Institutional Theory. En S. R. Clegg, C. Hardy y W. Nord (eds.), Handbook of Organization Studies (pp. 175-190). Sage.

Xunta de Galicia (2008). Mapa galego de equipamentos e servizos sociais: Criterios para a planificación territorial e sectorial. Vicepresidencia da Igualdade e do Benestar.

Wildawsky, A. (1979). Speaking Truth to Power. The Art and Craft of Policy Analysis. Little Brown. 